\title{
Extracciones e indicaciones de extracciones dentales en población rural chilena de 11 a 30 años
}

\author{
Olate $\mathrm{S}^{*}$, Alister JP**, Soto $\mathrm{M}^{*}$, Alveal R*, Fuentes J*, Thomas D*
}

\section{RESUMEN}

Introducción: A pesar de la creciente tecnología odontológica y el progresivo aumento de la cantidad de odontólogos, el precario estándar de salud oral de la población rural se ha mantenido a lo largo del tiempo. El objetivo de esta investigación es describir y cuantificar las exodoncias de piezas dentarias permanentes en población rural. Metodología: Se realizó un estudio de tipo descriptivo seleccionando el $100 \%$ de los sujetos atendidos en el Consultorio Chol-Chol, (IX Región) de 11 a 30 años de edad que obtuvieron su alta integral durante los años 2001 y 2003. Los diagnósticos fueron realizados por dos odontólogos experimentados en ausencia de apoyo radiográfico (procedimiento realizado de acuerdo al instrumental y equipos disponibles). Se evaluó el estado de cada una de las piezas dentarias exceptuando los terceros molares.

Resultados: Fueron analizadas 181 fichas clínicas de los cuales el 36,42\% fueron del sexo masculino, siendo la edad media de la muestra 18,27 años. 143 sujetos presentaron piezas perdidas en el momento del examen, a 117 sujetos se les indico extracción de piezas dentales permanentes. Al finalizar el tratamiento 167 (92,2\%) sujetos presentaron ausencia de piezas dentarias permanentes, con un promedio de 5,6 piezas al finalizar el alta integral. Conclusión: Los pacientes adolescentes y adultos jóvenes en condiciones de ruralidad presentan alta frecuencia de patologías orales que determinan la exodoncia de las piezas dentales. Estos pacientes necesitaran a corto plazo extensas rehabilitaciones para recuperar su sistema estomatognático.

Palabras clave: Salud Oral, Ruralidad, Piezas dentales perdidas.

\section{SUMMARY}

Introduction: In spite of high end dental technology and the progressive increase in the amount of Dentists in our Country, the precarious standard of oral health in the rural population have stayed throughout the time, specially in ethnic groups such as Mapuche population. The objective of the present study is to describe and to quantify dental extractions in permanent dentition in rural population.

Methodology: A descriptive study was performed, selecting the $100 \%$ of subjects who received dental attention at "Consultorio Chol-Chol", (IX Region, Chile). The sample in this research were formed by subjects between 11 and 30 years. All of them received complete dental attention during years 2001 to 2003. The diagnoses were made by two experienced dentists in absence of $\mathrm{x}$-ray support (procedure made according to instruments and equipment available). The condition of each dental piece was evaluated excepting wisdom teeth.

Results: 181 clinical card files were analyzed; $36.42 \%$ were male with average age of the sample of 18.27 years. 143 subjects presented absence of one or more teeth at the time of the examination, 117 subjects were treated with dental extractions as part of the complete therapy. At the end of the dental treatment $167(92,2 \%)$ subjects presented absence of permanent teeth, with an average of 5.6 teeth when dental treatment was completed.

Conclusion: For the sample composed by adolescents and young adults the lack of oral health reported an absence and loss of teeth. Further complex rehabilitations of their dentures must be performed.

Key words: Oral Health, Rurality, Tooth loss. 
Aceptado para publicación: 2006.

* Departamento de Odontología Integral, Facultad de Medicina, Universidad de La Frontera, Temuco, Chile. ** Alumno Pre-Grado Carrera de Odontología Universidad de La Frontera, Temuco, Chile.

Olate S, Alister JP, Soto M, Alveal R, Fuentes J, Thomas D. Extracciones e indicaciones de extracciones dentales en población rural chilena de 11 a 30 años. Av. Odontoestomatol 2006; 22-2: 119-124.

\section{INTRODUCCIÓN}

La salud humana se presenta como un mosaico de diferentes situaciones donde interactúan los mas diversos factores. Sin embargo, existe consenso mundial en que el alto numero de patologías crónicas son las complicaciones mas importantes dada las diferentes implicancias que conlleva, como por ejemplo en el empobrecimiento de la calidad de vida, el impacto en la sociedad por los costos que demanda a los programas de salud, las limitaciones económicas que eventualmente sufren los pacientes que las padecen, las complicaciones en los tratamientos y la toma de decisiones, etc. Las caries y las periodontopatías son las manifestaciones mas claras en el ámbito oral, determinando diversas situaciones en su presentación, tratamiento y pronóstico.

A pesar de las enormes diferencias que existen entre ambas enfermedades, existen características comunes que las hacen aun más complejas, como la cronicidad de la patología, la etiología multifactorial, la necesidad de controles estricto para que los tratamientos conservadores tengan un buen pronóstico, la necesidad de cambios de hábitos por parte de nuestros pacientes y las obligaciones que recaen sobre los odontólogos para el mejoramiento de las técnicas de control y tratamiento de las enfermedades. Por otra parte las consecuencias de la falta de tratamiento o tratamientos defectuosos consiguen el aumento de la complejidad de los casos además del aumento del tiempo y costo de los tratamientos (1).

Los tratamientos destinados al control de la enfermedad son variados y depende de muchos factores. Uno de los tratamientos entregados con cierta frecuencia a la población son las exodoncias, donde se remueve el "elemento perturbador" de la boca, y donde también se mutila al paciente eliminando una parte vital para el funcionamiento del sistema estomatognático que incluye la masticación, deglución, nutrición, fonación, convivencia social, etc.

Tal estilo de tratamiento se presenta como la solución a las complejas patologías orales en su estado terminal; no obstante, cuando el tratamiento indicado no se puede realizar (por motivos económicos en la mayoría de los casos), y aunque sea en las fases iniciales y recuperables de la patología, se indica la exodoncia de la pieza dentaria (2).

A pesar de la evolución de la tecnología en odontología, del mejoramiento de las técnicas para la conservación dentaria y del constante aumento del recurso humano odontológico, las exodoncias parece ser que se realizan con bastante mas frecuencia de lo que creemos. En la selección de este tipo de tratamiento, sin duda alguna tiene un gran importancia la situación socioeconómica del paciente, toda vez que las patologías orales mencionadas se encuentran dentro de las 4 enfermedades de tratamiento más costoso en el mundo (2). Especial atención debemos entregar a la población de escasos recursos y del ámbito rural, ya que en la mayoría de los casos, presentan menos acceso a la atención odontológica.

El objetivo del presente estudio es cuantificar las exodoncias e indicaciones de extracción realizadas en población rural.

\section{MATERIAL Y MÉTODOS}

Se desarrollo un estudio de tipo descriptivo en el Consultorio Chol - Chol de la comuna del mismo nombre (el estudio se desarrollo cuando la localidad 
de Chol - Chol era una zona rural, parte de la comuna de Nueva Imperial, IX región. La comuna de Chol - Chol existe como tal solamente desde el año 2005).

El estudio se desarrollo revisando todas las fichas clínicas de pacientes atendidos en dicho Consultorio desde los años 1997 al 2001. De ellos se selecciono al $100 \%$ de los pacientes de entre 11 y 30 años.

Con estas fichas se completo una ficha de registro en la que se encontraba los datos personales del individuo, las exodoncias realizadas y las indicaciones de exodoncias en su examen y diagnóstico inicial. Se descarto la evaluación a los terceros molares.

Las indicaciones de exodoncia se realizaron en base a algunos criterios definidos por los examinadores, como:

1. Presencia de caries extensa con posibilidad de rehabilitación nula o escasa por medio de técnica directa.

2. Presencia de periodontitis avanzada con perdida de inserción mayor a $1 / 2$ de la longitud radicular promedio según la pieza dentaria.

3. Pulpitis irreversible, necrosis pulpar y comunicaciones de la cámara pulpar con el medio externo en molares y premolares.

A través de tablas dinámicas realizadas en el programa computacional Microsoft Excel se ordenó los datos y se obtuvo información para cada sujeto y para cada pieza dentaria. Se identifico la media de exodoncias e indicaciones de exodoncia por pacientes, cuales son las piezas extraídas con más frecuencia, necesidad de tratamientos rehabilitadores, etc.

\section{RESULTADOS}

181 fichas clínicas fueron incluidas en el estudio. Este número corresponde al $100 \%$ de los pacientes de entre 11 y 30 años que obtuvieron tratamiento y alta integral en el Consultorio Chol - Chol. Los diagnósticos fueron realizados por dos odontólogos con más de 2 años de experiencia que trabajaban en el Consultorio Chol - Chol. El procedimiento diagnóstico se realizó con instrumental de examen (explorador, espejos dentales, pinzas, etc.) en ausencia de apoyo radiográfico o de exámenes de laboratorios.
Setenta y un sujetos fueron hombres $(36,42 \%)$ con una edad media de 18,06 años, mientras que 110 $(63,58 \%)$ pacientes fueron mujeres con una edad media de 18,18 años. Un total de 687 piezas dentales permanentes fueron extraídas al momento del diagnostico, mientras que 328 presentaron indicación de exodoncia.

Para fines del estudio, los pacientes se distribuyeron en 5 grupos, donde el criterio que primó fue la edad de los pacientes; la tabla 1 muestra la distribución de la muestra. Se aprecia que en el grupo de las mujeres, el $46,36 \%$ tuvo una edad entre 15 y 18 años, mientras que en el grupo de los hombres, el 36,6\% se presento en el mismo grupo etéreo.

La tabla 2 muestra el estado de las piezas al momento del examen y diagnostico. Ella muestra que casi el $13,6 \%$ de las piezas dentaria esta perdida al momento del diagnóstico odontológico, mientras que el 6,5 \% se presenta con indicación de extracción para alguna de las piezas dentarias. Esto señala que al momento del alta integral del paciente, el 20,03\%, es decir, 5,6 dientes se encuentran perdidos.

Además, a medida que la edad avanza, también lo hacen la perdida de piezas; de esta forma, a los 14 años casi 3 piezas dentales se encuentran perdidas mientras que a los 30 años, sólo el 59,18\% de las piezas dentarias se encuentran sanas u obturadas, siendo un total de 11,42 las piezas dentales perdidas en dicho grupo de edad. Esto muestra que en el transcurso de 16 años (desde 14 años a los 30 años) se pierden 8,42 piezas dentarias.

La tabla 3 muestra la frecuencia de exodoncias según grupo dentario. Se aprecia que el grupo perdido con mas frecuenta es el de los molares inferiores, le siguen los molares superiores, premolares

\begin{tabular}{|lccccc|}
\hline \multicolumn{5}{|c|}{ TABLA 1.- SEXO V/S EDAD } \\
DE LA MUESTRA \\
\hline Sexo & Edad 1 & Edad 2 & Edad 3 & Edad 4 & Edad 5 \\
& $11-14$ & $15-18$ & $19-22$ & $23-26$ & $27-30$ \\
\hline Femenino & 21 & 51 & 24 & 12 & 7 \\
Masculino & 11 & 26 & 17 & 5 & 7 \\
\hline
\end{tabular}




\begin{tabular}{|c|rrcc|}
\hline \multicolumn{5}{|c|}{ TABLA 2.- ESTADO DE PIEZAS DENTALES POR GRUPO DE EDADES } \\
\hline \multirow{2}{*}{$\begin{array}{c}\text { Rango } \\
\text { de } \\
\text { edad }\end{array}$} & \multicolumn{4}{|c|}{ Estado de piezas dentales } \\
\cline { 2 - 5 } $\mathbf{1}(11-14)$ & Presente & Ausente & Ind. Exod. & Piezas perdidas al alta integral \\
$\mathbf{2}(15-18)$ & $89,29 \%$ & $6,81 \%$ & $3,91 \%$ & 2,99 \\
$\mathbf{3}(19-22)$ & $78,77 \%$ & $9,74 \%$ & $6,49 \%$ & 4,54 \\
$\mathbf{4}(23-26)$ & $66,60 \%$ & $14,46 \%$ & $7,32 \%$ & 6,09 \\
$\mathbf{5}(27-30)$ & $59,18 \%$ & $29,94 \%$ & $5,46 \%$ & 9,35 \\
\hline Total & $\mathbf{7 9 , 9 7 \%}$ & $\mathbf{1 3 , 5 6 \%}$ & $\mathbf{6 , 4 7} \%$ & $5,47 \%$ \\
\hline
\end{tabular}

\begin{tabular}{|ccccc|}
\hline \multicolumn{5}{|c|}{ TABLA 3.- FRECUENCIA DE EXODONCIA SEGÚN GRUPO DENTARIO } \\
\hline Grupo de piezas & Presente & Ausente & Ind. Exod. & Piezas perdidas al alta integral \\
\hline M inf & $49,72 \%$ & $36,74 \%$ & $13,54 \%$ & 2,01 \\
M sup & $63,67 \%$ & $25,41 \%$ & $10,91 \%$ & 1,45 \\
PM sup & $70,44 \%$ & $18,92 \%$ & $10,64 \%$ & 1,18 \\
PM inf & $90,06 \%$ & $5,80 \%$ & $4,14 \%$ & 0,39 \\
Ant sup & $90,98 \%$ & $5,16 \%$ & $3,87 \%$ & 0,54 \\
Ant inf & $99,63 \%$ & $0,18 \%$ & $0,18 \%$ & 0,02 \\
\hline Total & $\mathbf{7 9 , 9 7 \%}$ & $\mathbf{1 3 , 5 6 \%}$ & $\mathbf{6 , 4 7 \%}$ & 5,59 \\
\hline
\end{tabular}

superiores y premolares inferiores. Los terceros molares fueron excluidos del estudio; de esta forma, de los 4 molares inferiores, 2 se encuentran perdidos al alta integral y, de los 4 molares superiores, 1,5 se encuentran perdidos. Los primeros molares permanentes, insertos dentro de los grupos molares, se pierden con relativa homogeneidad; la tabla 4 muestra la prevalencia de la perdida dentaria del

\begin{tabular}{|c|c|c|c|c|c|c|c|c|}
\hline \multicolumn{9}{|c|}{$\begin{array}{l}\text { TABLA 4.- PRIMEROS MOLARES } \\
\text { PERMANENTES PERDIDOS }\end{array}$} \\
\hline & \multicolumn{2}{|c|}{1,6} & \multicolumn{2}{|c|}{2,6} & \multicolumn{2}{|c|}{3,6} & \multicolumn{2}{|c|}{4,6} \\
\hline & $\mathrm{n}$ & $\%$ & $n$ & $\%$ & $\mathrm{n}$ & $\%$ & $\mathbf{n}$ & $\%$ \\
\hline $\begin{array}{l}\text { Pieza } \\
\text { ausente }\end{array}$ & 113 & 62,4 & 102 & 56,4 & $130^{\circ}$ & 71,8 & 121 & 66,8 \\
\hline
\end{tabular}

primer molar para la muestra; siempre los molares inferiores se pierden con mas frecuencia.

La tabla 5 muestra la relación existente entre la ausencia dentaria al momento del examen y las indica-

\begin{tabular}{|ccc|}
\hline \multicolumn{3}{|c|}{ TABLA 5.- RELACIÓN ENTRE PIEZAS } \\
AUISENTES E INDICACIÓN DE EXODONCIA \\
\hline Rango de Edad & Ausente & Ind. Exod. \\
\hline $\mathbf{1}(11-14)$ & 1 & 0,56 \\
$\mathbf{2}(15-18)$ & 1 & 0,66 \\
$\mathbf{3}(19-22)$ & 1 & 0,51 \\
$\mathbf{4}(23-26)$ & 1 & 0,20 \\
$\mathbf{5}(27-30)$ & 1 & 0,37 \\
\hline Total & $\mathbf{1}$ & $\mathbf{0 , 4 8}$ \\
\hline
\end{tabular}


Olate S, Alister JP, Soto M, Alveal R, Fuentes J, Thomas D. Extracciones e indicaciones de extracciones dentales en población rural chilena de 11 a 30 años

ciones de exodoncias respectivas; se aprecia que por cada pieza perdida en la muestra existe casi 0,5 piezas con indicación de exodoncia. Se aprecia además que los grupos 1, 2 y 3 presentaron la mayor relación entre piezas perdidas e indicaciones de exodoncia.

\section{DISCUSIÓN}

181 fichas clínicas fueron evaluadas en pacientes que obtuvieron su alta integral. La edad de la población fluctuó entre los 11 y los 30 años y fue mayoritariamente del sexo femenino. La edad media de la población alcanzo a los 18 años.

Chol - Chol hasta el año 2004 era una localidad de la comuna de Nueva Imperial, distante $20 \mathrm{Km}$ al este de la comuna y $25 \mathrm{Km}$ al sur de la comuna de Temuco, capital de la IX Región. La localidad es de una importante concentración mapuche, con un nivel socioeconómico bajo a medio en la mayoría de los casos; además las viviendas se presentan en reducciones mapuches y el complejo urbano de la localidad de Chol - Chol.

Las características generales de la población estudiada revelan un déficit en varias áreas de la vida, donde la situación de salud es un aspecto importante. En tal dirección, la concepción de salud de los pobladores rurales está ligada con lo estrictamente curativo. La resignación evidenciada con respecto a la enfermedad los lleva a asumir una actitud pasiva hacia la misma; aparentemente, la enfermedad es una especie de designio que pareciera no poderse evitar (3). Las condiciones de salud oral es el resultado de la suma y resta de un verdadero mosaico de situaciones, donde las variables como entorno familiar, condicionantes de vida, educación del paciente y su entorno familiar, etc. cumplen un rol trascendental (4).

Jara (5) reveló ciertas características de la población mapuche en torno al proceso de salud y enfermedad. En ellas destaca la precaria condición oral con un COPD "muy alto" (según la OMS), progresiva introducción de alimentos azucarados y desconocimiento de los principales agentes etiopatogénicos de la caries; además, población adulta y de la tercera edad identificaba la perdida de piezas dentarias como consecuencias de factores medio ambientales (pas- mos y aires). En estas comunidades, entre el $46 \%$ y el $64 \%$ tiene cepillo dentario; como alternativas al cepillado utilizan pasta dental sola (34\%), bicarbonato (19\%), solo el dedo o nada (23\%). Sólo un 58\% utiliza pasta dental. El tratamiento odontológico entregado con mayor frecuencia fue la extracción dentaria, variando la perdida dentaria desde solo una pieza hasta la completa perdida dentaria.

Las principales causas de perdida dentaria son las caries y las periodontopatías; en la IX región, la presencia de caries en la población infantil entre 6 y 8 años es superior al $90 \%$ (6, 7 y 8$)$.

La tendencia actual en los países con fuertes políticas de salud es la disminución de los indicadores de caries, favoreciendo la inversión en recursos destinados al mejoramiento del recurso humano y tecnológico; además, la motivación y el autocuidado juegan un rol muy importante (2). De esta forma, en países europeos como Suiza los niveles de salud oral indican disminución en los índices de caries bajando progresivamente desde 13,89 a 1,61 en pacientes de 15 años de edad, donde el uso de fluoruros a jugado un rol importante (9).

En nuestro estudio, evidenciamos un fuerte daño al sistema estomatognático, con una perdida dentaria muy alta y progresiva con la edad; así, a los 18 años (edad promedio de la muestra) existe casi 6 piezas dentaria perdidas por paciente, donde el principal grupo perdido es el de los molares inferiores, seguido muy de cerca por los molares superiores. Al finalizar el tratamiento, cerca del $92 \%$ de los pacientes se encuentra con piezas dentales perdidas.

Según Gamonal (10), en la Región Metropolitana (región donde se encuentra la capital de Chile) existe una perdida dentaria cercana a las 10,34 en adultos de 35 a 44 años, lo cual es menor a las 11,42 piezas perdidas en pacientes de 27 a 30 años de nuestra muestra.

Según la Encuesta Nacional de Salud (11) realizada en Chile, en pacientes de 16 a 24 años solo $61,8 \%$ de los pacientes presenta dentición completa. Mapuches de 14 a 30 años presentan un índice COP$\mathrm{D}$ de 18,15 (12). El alto nivel de estas cifras se condiciona con el nivel patológico que existe en la infancia de la población; así, en pacientes de 12 años 
existe 0.38 piezas permanentes perdidas nivel nacional, mientras que en la IX Región existe 0,69 piezas perdidas. En nuestro estudio en pacientes de 11 a 14 existe un promedio de 2,99 piezas perdidas lo que representa 9 veces más que para el país (11). Finalmente, las relaciones de la ruralidad con la salud han sido históricamente más curativa que preventiva, lo cual indica un difícil camino en la lucha de la prevención en odontología (13).

\section{CONCLUSIÓN}

Existe un alto daño del sistema estomatognático en población rural, con una fuerte perdida dentaria a corta edad. La perdida dentaria aumenta progresivamente con los años de vida y existe una importante relación entre la ausencia dentaria y la indicaciones de exodoncia. Esta situación implica una alta necesidad de futuras rehabilitaciones lo que conlleva importantes costos en el tratamientos de estos pacientes.

\section{BIBLIOGRAFÍA}

1. Yee R, McDonald N. Caries experience of 5-6year-old and 12-13-year-old schoolchildren in central and western Nepal. Int Dent J. 2002; 52(6):453-60.

2. Petersen PE, The world oral health 2003: continuous improvement of oral health in the $21^{\text {st }}$ century - the approach of the WHO Global oral Health Programme; Community Dent Oral Epidemiol 2003; 31 (suppl. 1):3-24

3. Hilas E, Tessio Conca A, Moncunill I, Cornejo LS. Concepciones de salud predominantes en comunidades rurales dispersas. Rev Cubana Estomatol 2004; 41(3): p.0-0. ISSN 0034-7507.

4. Newton JT, Bower E. The social determinants of health: new approaches to coneptualizing and researching complex causal networks. Community Dent Oral Epidemiol 2005; 33: 25-34.

5. Jara D, Moya A. Conductas, valores y creencias de dos comunidades mapuches en relación con su salud oral. Universidad de La Frontera, 1997.
6. Gonzalez, Henrriquez. Prevalencia de carie según índice COPD y ceod en escolares entre seis y ocho años de la comuna de Nueva Imperial IX región Chile. Temuco, Universidad de La Frontera, 1998.

7. Hermosilla M, Azocar C. Estudio descriptivo de la correlacion de los indices COPD y ceod en escolares básicos de la comuna de Temuco. Temuco, Universidad de La Frontera, 2000

8. Hinostroza M, Frantzen J. Prevalencia de caries en escolares mapuches y no mapuches de la comunad e Tolten IX Region. Universidad de La Frontera, 1997.

9. Marthaler T, Menghini G, Steiner M. Use of the Significant Caries Index in quantifying the changes in caries in Switzerland from 1964 to 2000. Community Dent Oral Epidemiol 2005;33:159-66.

10. Gamonal J, López N, Aranda W. Periodontal conditions and treatment needs, by CPITN, in the 35-44 and 65-74 years old population in Santiago, Chile. Int Dent J 1998;48:96-103

11. Encuesta Nacional de Salud 2003. Ministerio de Salud de Chile, 2004.

12. de la Maza FJ, Cueto MV. Estudio epidemiológico de la salud oral en una población mapuche adulto joven . Odontol Chil. 1989; 37(1):183-5

13. Espinosa L, Cambios del modo y estilo de vida; su influencia en el proceso salud-enfermedad. Rev Cubana Estomatol 2004; 41 (3): p.0-0. ISSN 0034-7507.

\section{CORRESPONDENCIA}

Sergio Olate Morales

Departamento de Odontología Integral

Facultad de Medicina

Universidad de La Frontera,

Manuel Montt 112, Piso $4^{\circ}$.

Temuco, Chile

eMail:solat001@hotmail.com,

sergio.olate.morales@gmail.com 